\title{
AMBIENTE DE INOVAÇÃO: ESTUDO COMPARATIVO ENTRE TRÊS UNIDADES DE UMA ORGANIZAÇÃO DO SETOR METAL-MECÂNICO
}

\author{
Luciano Castro de Carvalho - EAESP/FGV \\ Denise Del Pra Netto Machado - FURB ${ }^{2}$
}

Resumo: Conhecer os fatores motivacionais ao desenvolvimento dos processos de inovação tem despertado interesse na área de estudos organizacionais, sendo um desafio para o campo da Gestão. Nessa linha, o presente trabalho buscou identificar as semelhanças/diferenças nas características do ambiente inovador nas três unidades da empresa que ganhou por dois anos consecutivos o prêmio FINEP de inovação, e ainda verificar se as características percebidas por esses ambientes possuem aderência ao modelo proposto pelo Minnesota Innovation Research Program (MIRP) com base na metodologia Minnesota Innovation Survey (MIS). Foram pesquisados 349 funcionários dispersos geograficamente entre as três filiais localizadas nos estados do Rio Grande do Sul, Goiás e São Paulo. Os sujeitos sociais responderam a um questionário adaptado do modelo proposto, contendo três grupos de dimensões que envolvem características internas, externas e de resultados, totalizando 21 dimensões e englobando 45 questões com opções de resposta em escala Likert de 5 pontos. Foi utilizado o alfa de Cronbach, análise de frequência e a modelagem de equações estruturais como ferramentas de análise. Como resultado, apurou-se que das 21 dimensões estudadas, 10 foram percebidas de forma comum nas três unidades como constantes de um ambiente favorável ao desenvolvimento de inovações e 7 não foram percebidas como existentes no ambiente estudado. No total das 21 dimensões, 17 obtiveram percepções comuns, embora em frequência diferentes. Verificou-se nas três unidades que fator que caracteriza o ambiente de inovação refere-se à participação dos empregados no processo de tomada de decisão sobre inovações. De forma geral, pode-se afirmar que as unidades possuem mais semelhanças do que diferenças, e que a unidade de Goiás teve melhor adesão ao modelo proposto pelo MIRP devido às correlações existentes entre dimensões internas e externas sobre a percepção de eficiência da inovação. Assim, conforme a metodologia MIS, as unidades de Goiás e Rio Grande do Sul apresentam evidências de ser a mais e menos inovadora, respectivamente.

Palavras-chave: Ambiente de Inovação; Minnesota Innovation Survey (MIS); Gestão da Inovação.

\footnotetext{
${ }^{1}$ E mail: luccar@gmail.com

2 E mail: delpra@furb.br. Endereço: Rua Antônio da Veiga, 140 - Victor Konder CEP $89012-$ 900 - Blumenau - SC.
}

CARVALHO, L. C.; MACHADO, D.D.P.N. Ambiente de Inovação: estudo comparativo entre três unidades de uma organização do setor metal-mecânico. Revista de

Empreendedorismo e Gestão de Pequenas Empresas, v. 2, n.1, p. 47-76, 2013. 


\title{
ENVIRONMENT OF INNOVATION: COMPARATIVE STUDY OF THREE UNITS OF AN ORGANIZATION OF METAL-MECHANICAL INDUSTRY
}

\begin{abstract}
Knowing the motivating factors to the development of innovation processes has aroused interest in the area of organizational studies, is a challenge for the field of management. In this line, the present study aimed to identify the similarities/differences in the characteristics of the innovative environment in the three units of the company for two consecutive years won the FINEP prize for innovation, and verify that the perceived characteristics of these environments have adherence to the model proposed by Minnesota Innovation Research Program (MIRP) based on the methodology Minnesota Innovation Survey (MIS). In total, 349 employees were surveyed which are dispersed geographically among three branches located in the states of Rio Grande do Sul, Goiás and Sao Paulo. The social subjects answered a questionnaire adapted from the model proposed, containing three groups of dimensions involving internal and external characteristics and results, a totaling 21 dimensions and encompassing 45 questions with answer options on a Likert scale of 5 points. Therefore, this study is characterized as descriptive with quantitative method. We used the Cronbach's alpha as an indicator of the reliability of data and frequency analysis, map exploratory factor and structural equation modeling as analysis techniques. As a result, it was found that the 21 dimensions studied, 10 were perceived in common in the three units as a constant in the environment for the development of innovation and 7 were not perceived as existing in this study. A total of 21 dimensions, 17 obtained common perceptions, though in different frequency. It was found in the three units that factor that characterizes the environment of innovation refers to the participation of employees in decision-making on innovations. In general, it can be stated that the units have more similarities than differences and that the branch of Goiás has better adhesion to the model proposed by MIRP due to correlations of internal and external dimensions on the perception of efficiency and innovation. Thus, according to the methodology MIS, the units of Goiás and Rio Grande do Sul show evidence of being most and less innovative, respectively.
\end{abstract}

Keywords: Innovation Environment; Minnesota Innovation Survey (MIS); Innovation Management.

\section{Introdução}

Por um longo período de tempo, a competitividade entre organizações buscou na teoria econômica, principalmente sob a abordagem da concorrência perfeita, as explicações sobre as oscilações ambientais. Nessa perspectiva, nenhum agente econômico sozinho, no caso as organizações, é capaz de alterar ou influenciar os preços de mercado. Esses agentes sob as mesmas condições, produzindo bens com características e qualidades semelhantes e com propriedade das mesmas

CARVALHO, L. C.; MACHADO, D.D.P.N. Ambiente de Inovação: estudo comparativo entre três unidades de uma organização do setor metal-mecânico. Revista de

Empreendedorismo e Gestão de Pequenas Empresas, v. 2, n.1, p. 47-76, 2013. 
informações, impugnavam aos preços o principal elemento da competitividade (FAGERBERG; SRHOLEC; KNELL, 2007).

Schumpeter (1942), por sua vez, aponta algumas deficiências a respeito de tal simplificação. Para o autor, a verdadeira natureza da competitividade capitalista não está alocada à concorrência dos preços, mas sim na de novos produtos, tecnologia, fonte de recursos e tipos de processo. Portanto, sob uma abordagem schumpeteriana, a competitividade das organizações está vinculada às inovações que introduzem novidade ao mercado e que promovem o desenvolvimento econômico.

Sendo assim, o comprometimento da organização com a inovação pode ser alicerçado pela promoção de um ambiente inovador, conforme descrito por Tidd, Bessant e Pavitt (1997). Segundo eles, os gestores possuem papel fundamental para o estímulo de um ambiente inovador, visto que por meio deles é designado o estilo de liderança, comunicação, motivação, estímulo à criatividade e, sobretudo, abertura para participação dos empregados/colaboradores no processo de inovação. Os empregados/colaboradores precisam estar envolvidos para que se tenha êxito em cinco áreas fundamentais: estratégia, processo, recursos, organização e aprendizado (JONASH; SOMMERLATTE, 2001).

No intuito de descobrir a existência de fatores de um ambiente organizacional que contribua para o incremento acentuado no desenvolvimento de inovações, a presente pesquisa possui como problemática central a identificação de semelhanças/diferenças nas características do ambiente inovador, com base na metodologia MIS, em três unidades dispersas geograficamente de uma empresa ganhadora por dois anos consecutivos do prêmio FINEP de Inovação. A empresa em questão foi listada pela Revista Época como uma das cem melhores empresas para se trabalhar.

A base metodológica se encontra nos trabalhos do Minnesota Innovation Research Program (MIRP), um programa da Universidade de Minnesota nos Estados Unidos que caracterizou o ambiente de inovação e estudou suas diferenças por meio da metodologia denominada Minnesota Innovation Survey (MIS). Esta 
metodologia oferece subsídios para a averiguação de dimensões que possam caracterizar o ambiente inovador das organizações.

A identificação das características dos ambientes das três unidades lotadas nos estados do Rio Grande do Sul, Goiás e São Paulo dará subsídios para a verificação da aderência destas unidades ao modelo proposto pelo MIRP para definição de um ambiente favorável a inovação.

\section{Referencial Teórico}

Neste capítulo são apresentados os principais conceitos teóricos que norteiam o estudo proposto. Para tanto, aborda-se o conceito de inovação e de ambiente favorável à inovação e sua caracterização.

\section{Inovação}

Como passar do tempo, o tema inovação ganha cada vez mais espaço em discussões e projetos universitários. Embora o tema seja abrangente e estudado sob várias perspectivas, as discussões não têm alcançado um direcionamento no que tange à definição de um conceito específico (DAMANPOUR, 1992).

Damanpour e Evan (1984) e Daft (1982), definem inovação como a adoção de uma ideia ou comportamento que pode ser representado por um sistema, política, programa, dispositivo, processo, produto ou serviço, não necessariamente inédito, mas que seja novo para a organização que o adota. Para Afuah (1999), a inovação está relacionada ao uso do conhecimento tecnológico e de experiências do mercado para ofertar novos produtos e serviços ao cliente.

Um dos conceitos mais utilizados em pesquisas sobre inovação, e até mesmo pela Pesquisa de Inovação Tecnológica (PINTEC), encontra-se no Manual de Oslo. Este manual, formulado pela Organização para Cooperação e Desenvolvimento Econômico (OCDE), propõe diretrizes para coleta e interpretação de dados sobre inovação tecnológica. Segundo o parágrafo 55 do Manual de Oslo (OECD, 2005), a inovação é caracterizada pelo implemento de um produto, bem ou serviço, ou 
processo novo ou substancialmente melhorado, ou ainda, novo método de marketing, novas práticas de negócios, nova organização do local de trabalho e novas relações externas.

Outra referência para os estudos sobre inovação encontra-se no Manual de Bogotá, elaborado pela Red Iberoamericana de Indicadores de Ciencia y Tecnología (RICYT) e pela Organización de Estados Americanos (OEA). O objetivo deste manual é de dispor de uma metodologia de medida e análise dos processos de inovação que facilite a comparabilidade entre países por meio de indicadores que são construídos para cada um deles e, ao mesmo tempo, detectar as especificidades das distintas idiossincrasias nacionais (RICYT, 2001)

Enquanto o foco original do Manual de Oslo remete ao conceito estrito de inovação, o Manual de Bogotá propõe captar as peculiaridades adotadas pelos processos de inovação e abarcar as estratégias empresariais que determinam os esforços tecnológicos das empresas. Não se trata de manuais excludentes, mas complementares.

Sendo assim, o presente estudo adota o conceito de inovação como sendo o desenvolvimento de novas práticas, processo, produto, gestão, design e negócios que podem gerar resultados novos ou substancialmente modificados para atender às expectativas organizacionais em resposta às demandas de mercado (DAMANPOUR, 1992; DAMANPOUR E EVAN, 1984; DAFT 1982; AFUAH, 1999; MANUAL DE OSLO, 2005; RICYT, 2001).

Outro ponto que merece destaque está na negligência da literatura em aceitar a inovação como um processo que apresenta vários estágios e com características específicas para cada um deles (DAMANPOUR, 1992). Esse processo é concebido por englobar o início, desenvolvimento e implemento de novas ideias ou comportamentos. Para tanto, admite-se que a inovação pode ser comprada ou desenvolvida pela organização, sendo que, em ambos os casos, a adoção da inovação está na expectativa de contribuir para o desempenho ou efetividade da organização e são respostas às mudanças nos ambientes interno e externo (DAMANPOUR, 1992).

CARVALHO, L. C.; MACHADO, D.D.P.N. Ambiente de Inovação: estudo comparativo entre três unidades de uma organização do setor metal-mecânico. Revista de

Empreendedorismo e Gestão de Pequenas Empresas, v. 2, n.1, p. 47-76, 2013. 


\section{Ambiente de Inovação}

Um dos grandes dilemas das teorias relacionadas à aprendizagem organizacional e criação do conhecimento é o entendimento de como as organizações traduzem os insights e conhecimento de seus colaboradores em conhecimento coletivo e habilidade organizacional (LAM, 2005). Enquanto Simon (1991) e Grant (1996) afirmam que a aprendizagem é basicamente uma atividade individual, a maioria das teorias destaca a importância da coletividade como recurso para o desenvolvimento das habilidades organizacionais (LAM, 2005). Neste contexto, conhecimento coletivo pode ser definido como aquele acumulado pela organização e armazenado em regras, procedimentos, rotinas e normas que servem como guia para o processo de resolução de problemas e padrão de interação entre seus membros. Pode ser ainda representado como o fluxo de conhecimento emergente das interações (LAM, 2005).

Tornatsky et al. (1983) destaca que embora existam estudos que procuram descrever os antecedentes (ambientes ou contextos sociais) ou os resultados da inovação (geração de conhecimento), poucos têm examinado "como" e "porque" as inovações emergem, desenvolvem, crescem ou terminam ao longo do tempo. $\mathrm{Na}$ tentativa de explicar os "comos" e "porques" do processo de inovação, Terra (1999) afirma que a teoria organizacional e as necessidades impostas pelo ambiente têm evoluído no sentido de promover uma crescente participação da contribuição intelectual dos trabalhadores e uma gestão proativa da criatividade, aprendizagem e do conhecimento.

Com vistas aos elementos apresentados, o meio inovador interno designa o contexto que está inserido todo o processo de inovação, incluindo liderança e gestão estratégica organizacional, capacidade de interpretação, interações com o ambiente externo e cultura organizacional (BARBIERI; ALVARES, 2005).

Para Álvares et al. (2003), as características do meio inovador interno e a forma com que as interações se desenvolvem com os demais ambientes podem definir o sucesso da inovação. Algumas das características do ambiente de inovação foram elencadas pelo Fórum de Inovação e inclui: gestão participativa, 
flexibilidade, valorização da aprendizagem, confiança, enfrentamento aberto de conflitos, tolerância ao erro, liberdade de opinião e expressão.

Os ambientes de inovação são mais inclusivos e abrangentes se comparados à perspectiva dos sistemas de inovação (ANDRADE, 2005). Nos sistemas de inovação, os elementos provenientes da herança cultural e da criatividade peculiar de um determinado grupo social não são reconhecidos como componentes da inovação, diferente do que ocorre nos ambientes de inovação.

Para Maciel (1997), o conceito de ambiente de inovação em estudos sociais sobre produção tecnológica:

[...] procura dar conta do conjunto de condições - limites, obstáculos, possibilidades, estímulos - da inovação em uma determinada formação social. Ambiente de inovação refere-se, portanto, ao conjunto de fatores políticos, econômicos, sociais e culturais que estimulam ou dificultam a inovação [...]. (MACIEL, 1997, p. 109).

Observa-se também nos trabalhos de Knox (2002), a menção de alguns elementos que podem manter uma organização como inovadora. $\mathrm{Na}$ opinião do autor, a cultura e clima organizacional, capacidades e habilidades de gerenciamento, controle e estrutura organizacional, novos produtos e desenvolvimento de processos têm o papel de manutenção da organização com vantagem competitiva sobre o mercado em decorrência da inovação gerada. Embora tenha destacado esses elementos como mantenedores de uma empresa inovadora, o autor relata que a inovação contínua está pautada no conhecimento e capacidades individuais de seus colaboradores. Para isto, faz-se necessário uma gestão que impulsione a geração de ideias inovadoras e empreendedoras, e ainda a liderança voltada a trabalhos em equipe.

\section{Metodologia}

A pesquisa está dentro dos moldes de um estudo descritivo com método quantitativo. As pesquisas descritivas podem apresentar as relações entre variáveis, 
expondo as características de determinada população ou fenômeno, mas não se atendo à explicação das características que descreve (VIEIRA, 2002).

Os planos da pesquisa descritiva se formam no intuito de medir características de um determinado construto teórico (HAIR Jr. et al., 2005). No caso da presente pesquisa, estas características se referem às dimensões de ambiente de inovação, ou seja, aquele que favorece o surgimento de inovações, utilizando-se para isto da metodologia MIS.

O período para realização da pesquisa, incluindo coleta de dados, foi de setembro de 2009 a janeiro de 2010, sendo o corte temporal transversal. Os dados transversais dão ao usuário um panorama dos elementos estudados em um dado ponto no tempo (HAIR Jr. et al., 2005). Tais dados foram coletados apenas uma vez durante o período de investigação, sintetizados e tratados estatisticamente.

A técnica utilizada foi survey com base no questionamento aos participantes sobre suas percepções acerca do ambiente de inovação. A empresa objeto de estudo, aqui denominada empresa Alpha, foi escolhida de forma intencional pelo fato de ser uma das empresas mais inovadoras no Brasil e ganhadora do prêmio FINEP em dois anos consecutivos. A pesquisa abrangeu as três filiais situadas nos estados de São Paulo (SP), Rio Grande do Sul (RS) e Goiás (GO).

A população de empregados nas três unidades totaliza 991. Deste total, foram considerados como sujeitos respondentes apenas aqueles ligados ao processo de inovação da empresa, seja em setor administrativo, de manutenção ou produção. A amostra por conveniência obteve grau de significância e erro amostral, calculados com base em Barbetta (2001), em que:

Tabela 1: Cálculo da Amostra das Unidades Pesquisadas.

\begin{tabular}{|c|c|c|c|c|}
\hline UNIDADE & População & $\begin{array}{c}\text { Erro } \\
\text { amostral }\end{array}$ & $\begin{array}{c}\text { Grau de } \\
\text { Significância }\end{array}$ & Amostra \\
\hline SP & 512 & $9,6 \%$ & $90,4 \%$ & 90 \\
\hline GO & 110 & $7,6 \%$ & $92,4 \%$ & 67 \\
\hline RS & 369 & $5,0 \%$ & $95,0 \%$ & 192 \\
\hline TOTAL & $\mathbf{9 9 1}$ & & & $\mathbf{3 4 9}$ \\
\hline
\end{tabular}

Fonte: Os Autores (2013).

CARVALHO, L. C.; MACHADO, D.D.P.N. Ambiente de Inovação: estudo comparativo entre três unidades de uma organização do setor metal-mecânico. Revista de

Empreendedorismo e Gestão de Pequenas Empresas, v. 2, n.1, p. 47-76, 2013. 
Os dados foram coletados por meio de questionário estruturado em conformidade com a metodologia MIS (Minnesota Innovation Survey), criada pelo Minessota Innovation Research Program (MIRP) da Universidade de Minessota nos Estados Unidos.

$O$ instrumento de coleta engloba 29 dimensões que caracterizam um ambiente inovador. Estas dimensões se encontram descritas nos estudos de Van de Ven e Poole (1990) e foram testadas no Brasil por Barbieri et al. (2003), Machado (2004), Vicenti (2006).

O questionário MIS criado pelo MIRP, foi desenvolvido originalmente em língua inglesa e possui duas partes: MIS I e MIS II, englobando 41 questões objetivas e 10 subjetivas. Essas questões tratam de fatores internos e externos à organização e que podem interferir no processo de inovação.

O questionário utilizado foi adaptado do modelo proposto pelo MIRP, deixando apenas as dimensões que poderiam ser perceptíveis aos funcionários da organização e não apenas à alta administração. Este questionário adaptado contemplou 21 dimensões agrupadas em três grandes grupos conforme a Tabela 2: 
Tabela 2: Estrutura do Questionário Adaptado do MIRP.

\begin{tabular}{|c|c|c|c|}
\hline Grupo & Dimensão & Nome da dimensão & Questões no questionário \\
\hline Resultados & 1 & Eficiência da inovação percebida & 1,2 e 3 \\
\hline \multirow[t]{8}{*}{$\begin{array}{l}\text { Dimensões } \\
\text { Internas }\end{array}$} & 2 & $\begin{array}{l}\text { Nível de incerteza que envolve a } \\
\text { inovação }\end{array}$ & $39,40,41,42$ e 44 \\
\hline & 3 & Escassez de recursos & $29 a, 29 b, 29 c, 29 d$ e 43 \\
\hline & 4 & $\begin{array}{l}\text { Padronização de procedimentos } \\
\text { para inovar }\end{array}$ & $31,33 a$ e $33 b$ \\
\hline & 5 & Grau de influência sobre decisões & 30a, 30b, 30c e 30d \\
\hline & 6 & Expectativas de prêmios e sanções & $4 a, 4 b, 5 a, 5 b$ \\
\hline & 7 & Liderança do time de inovação & $6,7,8,9,10$ e 17 \\
\hline & 8 & Liberdade para expressar dúvidas & 11,12 e 13 \\
\hline & 9 & Aprendizagem encorajada & 14,15 e 16 \\
\hline \multirow{4}{*}{$\begin{array}{l}\text { Dimensões } \\
\text { Externas }\end{array}$} & 10 & Dependência de recursos & 18 e 19 \\
\hline & 11 & Formalização no relacionamento & 20 e 21 \\
\hline & 12 & $\begin{array}{llll}\text { Eficiência percebida } & \text { com } & 0 \\
\text { relacionamento } & & \end{array}$ & 22,23 e 32 \\
\hline & 13 & Influência entre grupos & 24 \\
\hline \multirow[t]{2}{*}{$\begin{array}{l}\text { Dimensões } \\
\text { Internas }\end{array}$} & 22 & Frequência da comunicação interna & $\begin{array}{c}34 a, 34 b, 34 c, 34 d, 34 e, 34 f, \\
34 g\end{array}$ \\
\hline & 23 & Problemas identificados & $\begin{array}{c}37 \mathrm{a}, 37 \mathrm{~b}, 37 \mathrm{c}, 37 \mathrm{~d}, 37 \mathrm{e}, 37 \mathrm{f} \\
37 \mathrm{~g}, 37 \mathrm{~h}\end{array}$ \\
\hline \multirow{6}{*}{$\begin{array}{l}\text { Dimensões } \\
\text { Internas }\end{array}$} & 24 & Conflitos & 35 e $38 \mathrm{e}$ \\
\hline & 25 & Processos de resolução de conflitos & $38 a, 38 b, 38 c, 38 d$ \\
\hline & 26 & Complementaridade & 25 e 26 \\
\hline & 27 & Consenso/conflito & 27 e 28 \\
\hline & 28 & $\begin{array}{l}\text { Frequência da comunicação entre } \\
\text { grupos }\end{array}$ & 36 \\
\hline & 29 & Duração do relacionamento & 45 \\
\hline
\end{tabular}

Fonte: Os Autores (2013)

As questões possuem respostas em escala Likert de 5 pontos, sendo 10 valor de menor importância e 5 o de maior. O questionário proposto foi enviado eletronicamente para a área de recursos humanos da unidade de São Paulo, que distribuiu às demais unidades.

Vale lembrar que a adaptação do questionário desenvolvido pelo MIRP manteve os mesmos construtos, mas excluíram-se dimensões que não foram percebidas pelos empregados. Assim, a estrutura a ser verificada nos ambientes das unidades de RS, GO e SP contempla questões de dimensões internas, resultados e parte das dimensões externas. O uso de parte das dimensões externas justifica-se CARVALHO, L. C.; MACHADO, D.D.P.N. Ambiente de Inovação: estudo comparativo entre três unidades de uma organização do setor metal-mecânico. Revista de

Empreendedorismo e Gestão de Pequenas Empresas, v. 2, n.1, p. 47-76, 2013. 
por considerar àquelas dimensões externas ao grupo de inovação, mas que estão dentro da organização em estudo e que pudessem ser perceptíveis, ao passo que as dimensões excluídas referem-se a questões do ambiente externo que são mais percebidas pela alta administração. O modelo simplificado da relação entre estas dimensões, conforme Van de Ven e Chu (1989) apresenta-se na Figura 1.

Figura 1: Estrutura Adaptada da Metodologia MIS.

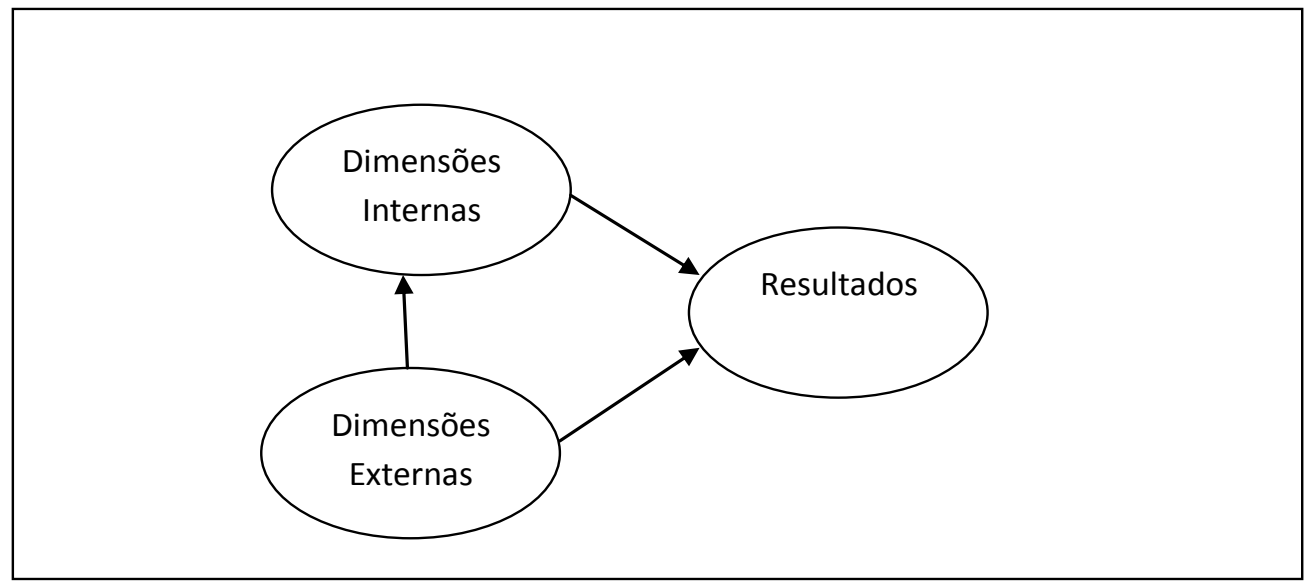

Fonte: Adaptado de Van de Ven e Chu (1989).

Entende-se que as dimensões externas que atuam como preditoras no modelo proposto, impactam nas características das dimensões internas, que por sua vez, impactam na percepção de eficiência nos resultados. Estes também são influenciados diretamente pelas dimensões externas.

Os dados obtidos por meio dos dois questionários adaptados foram tabulados em planilha do software Microsoft Excel. As perguntas foram agrupadas às dimensões as quais pertencem. Cada dimensão obteve uma média que correspondeu ao somatório das respostas das perguntas da dimensão por respondente e dividido pela quantidade de perguntas daquela dimensão.

A base de dados contida na planilha do Excel foi importada pelo software estatístico SPSS (Statistical Package for the Social Sciences) versão 17. A primeira análise realizada foi a de frequência, pela qual se pode evidenciar as respostas que apontaram as dimensões percebidas no ambiente inovador. Este procedimento foi 
realizado com as 45 questões do instrumento de coleta de dados, agrupadas em dimensões.

Após este procedimento foi realizado o teste de confiabilidade das respostas por meio do alfa de Cronbach. Este indicador aponta para o grau de convergência das respostas, ou seja, quanto menor a dispersão dos dados, maior a confiabilidade dos mesmos. Segundo Hair Jr. et al. (2005), este indicador, geralmente utilizado nas área de ciências sociais, é também conhecido como coeficiente de fidedignidade. As dimensões tiveram sua confiabilidade de respostas medidas e apresentadas em tabelas.

Em seguida, utilizou-se o mapa fatorial exploratório no intuito de verificar os agrupamentos das dimensões em fatores que poderiam representar o ambiente estudado (HAIR Jr et al., 2005). Foram realizados testes para identificação do número de fatores necessários, observando-se as cargas fatoriais das dimensões e as comunalidades entre elas. Após estes testes, verificou-se que três fatores seriam necessários e que melhor representariam os ambientes. Nesta análise, foram utilizadas apenas as dimensões internas percebidas pelos empregados.

A segunda análise buscou verificar se a estrutura da inovação proposta pelo MIRP, contendo dimensões internas, externas e resultados conforme a Figura 1, poderia ser observada nas unidades em estudo. Para isto, fez-se uso da técnica de modelagem de equações estruturais por meio do software estatístico SmartPLS 2.0, empregando as dimensões percebidas pelos funcionários. Este software permite gerar as equações de correlação entre as dimensões, demonstrando graficamente o valor de impacto e os valores para explicação do modelo (R2). A melhor aderência ao modelo proposto pelo MIRP pode ser evidenciada pela unidade que apresentar maior R2.

A comparação adequada entre os ambientes inovadores nas três unidades da organização estudada, mesmo que adotem políticas de gerenciamento da mesma natureza e com vários pontos em comum, pode ser afetada pelas especificidades de cada ambiente e idade de cada uma das unidades, além das características individuais de cada respondente.

CARVALHO, L. C.; MACHADO, D.D.P.N. Ambiente de Inovação: estudo comparativo entre três unidades de uma organização do setor metal-mecânico. Revista de

Empreendedorismo e Gestão de Pequenas Empresas, v. 2, n.1, p. 47-76, 2013. 


\section{Resultados}

O presente capítulo trata da identificação das características (dimensões) que podem representar os ambientes internos das três unidades de pesquisa. Para isto utilizou-se a análise de frequência de respostas para verificação da percepção das dimensões de um ambiente inovador. As dimensões percebidas pelos funcionários foram representadas pela letra "S" e as não percebidas pela letra "N". Para cada dimensão foi considerado o alfa de Cronbach como indicador de fidedignidade das respostas. Este índice, segundo Hair Jr. et al. (2005) indica o grau de convergência das respostas. Assim, quanto maior o índice, maior a fidedignidade das mesmas. Segundo o mesmo autor, índices superiores a 0,6 são considerados satisfatórios. A percepção dos empregados quanto às dimensões do ambiente inovador segue demonstrada na Tabela 3. 
Tabela 3: Percepção dos Funcionários Quanto às Dimensões do Ambiente Inovador.

\begin{tabular}{|c|c|c|c|c|c|c|c|}
\hline \multirow[b]{2}{*}{ Grupo } & \multirow[b]{2}{*}{ Dimensões } & \multicolumn{2}{|c|}{ Rio Grande do Sul } & \multicolumn{2}{|c|}{ Goiás } & \multicolumn{2}{|c|}{ São Paulo } \\
\hline & & $\begin{array}{c}\text { Percepçã } \\
0\end{array}$ & $\begin{array}{c}\text { Alfa de } \\
\text { Cronbac } \\
h\end{array}$ & $\begin{array}{c}\text { Percepçã } \\
0\end{array}$ & $\begin{array}{c}\text { Alfa de } \\
\text { Cronbac } \\
h\end{array}$ & $\begin{array}{c}\text { Percepçã } \\
0\end{array}$ & $\begin{array}{c}\text { Alfa de } \\
\text { Cronbac } \\
h\end{array}$ \\
\hline Resultado & $\begin{array}{l}\text { Eficiênc Inov } \\
\text { (1) }\end{array}$ & $\mathrm{S}$ & 0,75 & $\mathrm{~S}$ & 0,87 & $S$ & 0,88 \\
\hline \multirow{8}{*}{$\begin{array}{l}\text { Dimensõe } \\
\text { s Internas }\end{array}$} & $\begin{array}{c}\text { Incerteza Inov } \\
\text { (2) }\end{array}$ & $S$ & 0,35 & $S$ & 0,51 & $S$ & 0,46 \\
\hline & $\begin{array}{l}\text { Esc. Recursos } \\
\text { (3) }\end{array}$ & $\mathrm{N}$ & 0,86 & $\mathrm{~N}$ & 0,80 & $\mathrm{~N}$ & 0,84 \\
\hline & $\begin{array}{l}\text { Padron Proced } \\
\text { (4) }\end{array}$ & $S$ & 0,65 & $S$ & 0,77 & $S$ & 0,60 \\
\hline & $\begin{array}{c}\text { Influênc Decis } \\
\text { (5) }\end{array}$ & $\mathrm{S}$ & 0,76 & $S$ & 0,71 & $S$ & 0,69 \\
\hline & $\begin{array}{l}\text { Prêmio e Sanç } \\
\text { (6) }\end{array}$ & $\mathrm{N}$ & 0,51 & $\mathrm{~N}$ & 0,47 & $\mathrm{~N}$ & 0,48 \\
\hline & Liderança (7) & $S$ & 0,83 & $S$ & 0,91 & $S$ & 0,88 \\
\hline & $\begin{array}{l}\text { Liberd Exp Duv } \\
\text { (8) }\end{array}$ & $S$ & 0,69 & $S$ & 0,78 & $S$ & 0,69 \\
\hline & $\begin{array}{c}\text { Aprend } \\
\text { Encoraj (9) }\end{array}$ & $S$ & 0,70 & $\mathrm{~S}$ & 0,73 & $\mathrm{~S}$ & 0,70 \\
\hline \multirow{4}{*}{$\begin{array}{l}\text { Dimensõe } \\
\text { s Externas }\end{array}$} & $\begin{array}{c}\text { Depend } \\
\text { Recurs (10) }\end{array}$ & $S$ & 0,59 & $S$ & 0,64 & $S$ & 0,64 \\
\hline & $\begin{array}{c}\text { Formalização } \\
(11)\end{array}$ & $\mathrm{S}$ & 0,60 & $\mathrm{~S}$ & 0,34 & $S$ & 0,80 \\
\hline & $\begin{array}{c}\text { Eficien Perceb } \\
\text { (12) }\end{array}$ & $S$ & 0,66 & $S$ & 0,72 & $S$ & 0,76 \\
\hline & Influência (13) & $\mathrm{S}$ & 1,00 & $\mathrm{~S}$ & 1,00 & $S$ & 1,00 \\
\hline \multirow{8}{*}{$\begin{array}{l}\text { Dimensõe } \\
\text { s Internas }\end{array}$} & $\begin{array}{c}\text { Freq Com Inter } \\
\text { (22) }\end{array}$ & $S$ & 0,90 & $\mathrm{~N}$ & 0,92 & $\mathrm{~N}$ & 0,91 \\
\hline & $\begin{array}{c}\text { Problemas } \\
(23)\end{array}$ & $S$ & 0,89 & $S$ & 0,89 & $S$ & 0,91 \\
\hline & Conflitos (24) & $S$ & $-0,36$ & $S$ & 0,26 & $S$ & $-0,41$ \\
\hline & $\begin{array}{l}\text { Resol Conflito } \\
(25)\end{array}$ & $S$ & 0,15 & $S$ & $-0,06$ & $\mathrm{~S}$ & 0,20 \\
\hline & $\begin{array}{c}\text { Complement } \\
(26)\end{array}$ & $S$ & 0,56 & $S$ & 0,61 & $S$ & 0,79 \\
\hline & $\begin{array}{c}\text { Consenso/Con } \\
f(27)\end{array}$ & $S$ & 0,17 & $S$ & $-0,63$ & $S$ & 0,34 \\
\hline & $\begin{array}{c}\text { Freq Com Ext } \\
(28)\end{array}$ & $S$ & 1,00 & $S$ & 1,00 & $S$ & 1,00 \\
\hline & Duração (29) & $S$ & 1,00 & $\mathrm{~N}$ & 1,00 & $\mathrm{~S}$ & 1,00 \\
\hline
\end{tabular}

Fonte: Os Autores (2013) 
Conforme Tabela 3, as variáveis percebidas pelos empregados e que obtiveram índices de confiabilidade maiores que 0,6 relacionadas às dimensões internas, foram utilizadas no mapa fatorial para caracterização do ambiente inovador. As demais dimensões (externas e de resultados) juntamente com as dimensões de ambiente interno, foram utilizadas para a aplicação da modelagem de equações estruturais. São elas:

a) Rio Grande do Sul: dimensão 1, dimensão 4, dimensão 5, dimensão 7, dimensão 8 , dimensão 9, dimensão 11 , dimensão 12 , dimensão 13 , dimensão 23, dimensão 28 e dimensão 29.

b) Goiás: dimensão 1, dimensão 4, dimensão 5, dimensão 7, dimensão 8, dimensão 9, dimensão 10 , dimensão 12 , dimensão 13 , dimensão 23 , dimensão 26 e dimensão 28.

c) São Paulo: dimensão 1, dimensão 4, dimensão 5, dimensão 7, dimensão 8, dimensão 9, dimensão 10, dimensão 11, dimensão 12, dimensão 13, dimensão 23, dimensão 26, dimensão 28 e dimensão 29.

As variáveis que foram percebidas pelos funcionários, mas que obtiveram alfa de Cronbach abaixo de 0,6, podem indicar que os dados encontram-se dispersos e que, provavelmente, o índice para adequação ao ambiente de inovação foi obtido por aproximação. As dimensões não percebidas pelos funcionários e que obtiveram alfa de Cronbach elevado significam que as respostas realmente tendem a não visualização de tal dimensão no ambiente corporativo.

Após a identificação das variáveis, obteve-se o mapa fatorial, apontando três fatores ou dimensões que, conforme Van de Ven e Chu (1989) e ilustrado na Figura 1, podem caracterizar o ambiente inovador. O número de fatores levados em consideração teve por base testes que buscavam alcançar altas comunalidades e baixos valores específicos para o número de fatores.

$\mathrm{Na}$ filial do Rio Grande do Sul o agrupamento das dimensões em fatores comuns pode ser observado conforme Tabela 4.

CARVALHO, L. C.; MACHADO, D.D.P.N. Ambiente de Inovação: estudo comparativo entre três unidades de uma organização do setor metal-mecânico. Revista de

Empreendedorismo e Gestão de Pequenas Empresas, v. 2, n.1, p. 47-76, 2013. 
Tabela 4: Cargas Fatoriais para as Dimensões - Rio Grande do Sul.

\begin{tabular}{|c|c|c|c|}
\hline & \multicolumn{3}{|c|}{ Fatores } \\
\hline & 1 & 2 & 3 \\
\hline Pad Procec (4) &,- 017 & ,551 & 141 \\
\hline Influênc Dec (5) & , 103 & 686 & ,047 \\
\hline Liderança (7) & 695 & , 124 & ,036 \\
\hline $\begin{array}{l}\text { Liberd Expr } \\
\text { Duv (8) }\end{array}$ & ,772 & ,164 & ,017 \\
\hline $\begin{array}{l}\text { Aprend Encoraj } \\
\text { (9) }\end{array}$ & ,636 & ,087 & , 115 \\
\hline Problemas (23) & ,460 &,- 371 & 108 \\
\hline $\begin{array}{l}\text { Freq Com Ext } \\
(28)\end{array}$ & , 150 & ,443 & ,006 \\
\hline Duração (29) & 149 & 152 & 977 - r - \\
\hline
\end{tabular}

Fonte: Os Autores (2013)

Observa-se que no primeiro fator agruparam-se dimensões voltadas a liderança, ou seja, a liderança voltada para o time de inovação, a percepção de liberdade para expressar dúvidas, aprendizagem encorajada pelos gestores e ausência de barreiras para o desenvolvimento da inovação. Este fator representa a percepção dos empregados sobre a existência de um ambiente sem represálias para a expressão de suas dúvidas e opiniões.

O segundo fator se formou com dimensões relacionadas à padronização dos procedimentos, influência nas decisões e frequência de comunicação entre as partes do relacionamento voltado a inovação. A correlação entre estas variáveis exprime que a comunicação existente entre os grupos de inovação segue uma padronização ou protocolo e que esta interação pode resultar na influência do tipo de trabalho que será realizado pelas equipes. A interação entre grupos (dimensão 28) ocorre diariamente, na maioria das vezes, com ideias e opiniões verbalizadas. Neste fator, a dimensão referente ao grau de influência nas decisões pode ser evidenciada.

Já no fator 3, a dimensão constante se refere a duração do relacionamento entre os grupos de inovação (imensão 29). É notório que a confiança existente entre 
os grupos de inovação e a satisfação da parceria promove a percepção de duração do relacionamento mesmo após a implantação da inovação. O ambiente inovador da unidade localizada na região centro-oeste brasileira pode ser representado conforme Tabela 5.

Tabela 5:1 Cargas Fatoriais paras as Dimensões - Goiás.

\begin{tabular}{|l|r|r|r|}
\hline & \multicolumn{3}{|c|}{ Fatores } \\
\cline { 1 - 1 } & \multicolumn{1}{|c|}{1} & \multicolumn{1}{c|}{2} & \multicolumn{1}{l|}{3} \\
\hline Pad Procec (4) &, 203 &, 442 &,- 138 \\
Influênc Dec (5) &, 285 &, 900 &,- 100 \\
Liderança (7) &, 910 &, 019 &,- 089 \\
Liberd Expr Duv &, 840 &, 173 &, 252 \\
$\begin{array}{l}\text { (8) } \\
\text { Aprend Encoraj }\end{array}$ &, 574 &, 267 &, 428 \\
$\begin{array}{l}\text { (9) } \\
\text { Problemas (23) }\end{array}$ &, 130 &,- 224 &, 359 \\
$\begin{array}{l}\text { Complementar } \\
\text { (26) }\end{array}$ &, 726 &, 214 &, 343 \\
Freq Com Ext &,- 012 &, 450 &, 030 \\
$(28)$ & & & \\
\hline
\end{tabular}

Fonte: Os Autores (2013).

No fator 1, assim como na unidade do Rio Grande do Sul, foram agrupadas as dimensões relacionadas à liderança, mencionadas e acrescidas da dimensão "Complementaridade". Além da percepção das ações da liderança voltadas a um ambiente propício à exposição de ideias e dúvidas, com vistas ao aprendizado por meio do processo de inovação, os funcionários ainda acreditam que a interação com grupos de inovação promovem benefícios mútuos. Neste fator, a dimensão com maior carga fatorial foi atribuída à dimensão 7.

O fator 2 pode ser caracterizado por contemplar as dimensões relacionadas à padronização de procedimentos, grau de influência nas decisões e frequência de 
comunicação entre aos grupos. Neste fator, e de igual forma, na unidade do Rio Grande do Sul o grau de influência nas decisões é mais bem evidenciado.

A dimensão 23 (problemas identificados) caracteriza isoladamente o fator 3 . Entende-se que a ausência de problemas ou barreiras neste ambiente pode caracterizar esta organização. Observa-se que para a unidade de Goiás as cargas fatoriais são mais expressivas e com isso, o grau de correlação em relação a filial do Rio Grande do Sul é mais significativo.

A unidade de São Paulo apresenta uma maior concentração de dimensões no fator 1. A distribuição das dimensões internas percebidas pelos funcionários da unidade de SP pode ser visualizada por meio da Tabela 6.

Tabela 6:2 Cargas Fatoriais paras as Dimensões - São Paulo. Fonte: Os Autores.

\begin{tabular}{|l|r|r|r|}
\hline & \multicolumn{3}{|c|}{ Fatores } \\
\hline & \multicolumn{1}{|c|}{1} & \multicolumn{1}{l|}{2} & \multicolumn{1}{l|}{3} \\
\hline Pad Procec (4) &, 139 &, 531 &, 067 \\
Influênc Dec (5) &, 176 &, 687 &, 108 \\
Liderança (7) &, 687 &, 199 &, 388 \\
Liberd Expr Duv &, 795 &, 100 &, 101 \\
$(8)$ &, 742 &, 096 &,- 066 \\
Aprend Encoraj &, 353 &,- 397 &, 073 \\
$(9)$ &, 453 &, 240 &, 427 \\
Problemas (23) & & & \\
Complementar & & & \\
$(26)$ &,- 028 &,- 026 &, 589 \\
Freq Com Ext &, 221 &, 189 &, 279 \\
$(28)$ & & & \\
Complementar & & & \\
$(29)$ & &
\end{tabular}

Fonte: Os Autores(2013) 
O fator 1, assim como para as unidades do RS e GO, apresenta as dimensões ligadas à liderança (dimensões 7, 8 e 9). Ressalta-se que as ações da liderança nesta unidade estão ligadas também à percepção de complementaridade entre os grupos de inovação como geradoras de aditamentos recíprocos. Neste fator, não diferente das demais unidades, a percepção de um ambiente que favorece a expressão de ideias e dúvidas pode ser evidenciada.

No segundo fator, as dimensões referentes à padronização de procedimentos, influência nas decisões e ausência de barreiras para o surgimento de inovações podem ser verificadas. Entende-se que as regras e procedimentos na execução de tarefas ligadas a interação entre grupos, dá sustentação à percepção de grau de influência nas decisões. Verifica-se neste fator que o grau de influência nas decisões é característica marcante.

O terceiro fator pode ser representado pela dimensão referente à frequência de comunicação entre os grupos de inovação e complementaridade. Nele, a frequência de comunicação entre grupos pode ser destacada. No fator 2 evidenciouse que a padronização de procedimentos e a percepção de um elevado grau de influência nas decisões foram comuns nas três unidades. Pressupõe-se que assim como as unidades possuem lideranças semelhantes, as regras e métodos para execução das atividades seguem o mesmo protocolo. Esta suposição leva ao entendimento da existência de modelos padrões e unificados nas três unidades, já que os mesmos produtos e serviços são executados nas referidas unidades. Já o fator 3 não apresentou dimensões comuns nas três unidades.

Após a identificação das dimensões percebidas pelos funcionários, buscou-se verificar se a estrutura que deu origem a metodologia MIS (conforme a Figura 1), desenvolvida pelo MIRP e publicada por Van de Ven e Chu (1989) e Van de Ven e Poole (1990), são evidenciadas pelas organizações estudadas.

De acordo com a Figura 2, as dimensões externas na unidade do Rio Grande do Sul apresentam uma correlação maior entre elas se comparadas a dimensões internas. Esta dimensão tem correlação média-alta com as dimensões internas $(62,2 \%)$ e impacta diretamente os resultados em $25 \%$. Pode-se destacar a eficiência percebida na interação com outros grupos (dimensão12), a qual assegura

CARVALHO, L. C.; MACHADO, D.D.P.N. Ambiente de Inovação: estudo comparativo entre três unidades de uma organização do setor metal-mecânico. Revista de

Empreendedorismo e Gestão de Pequenas Empresas, v. 2, n.1, p. 47-76, 2013. 
um sentimento de confiança, responsabilidade e compromisso na realização das atividades em comum.

Figura 2: Estrutura da Metodologia MIS na Unidade do Rio Grande do Sul.

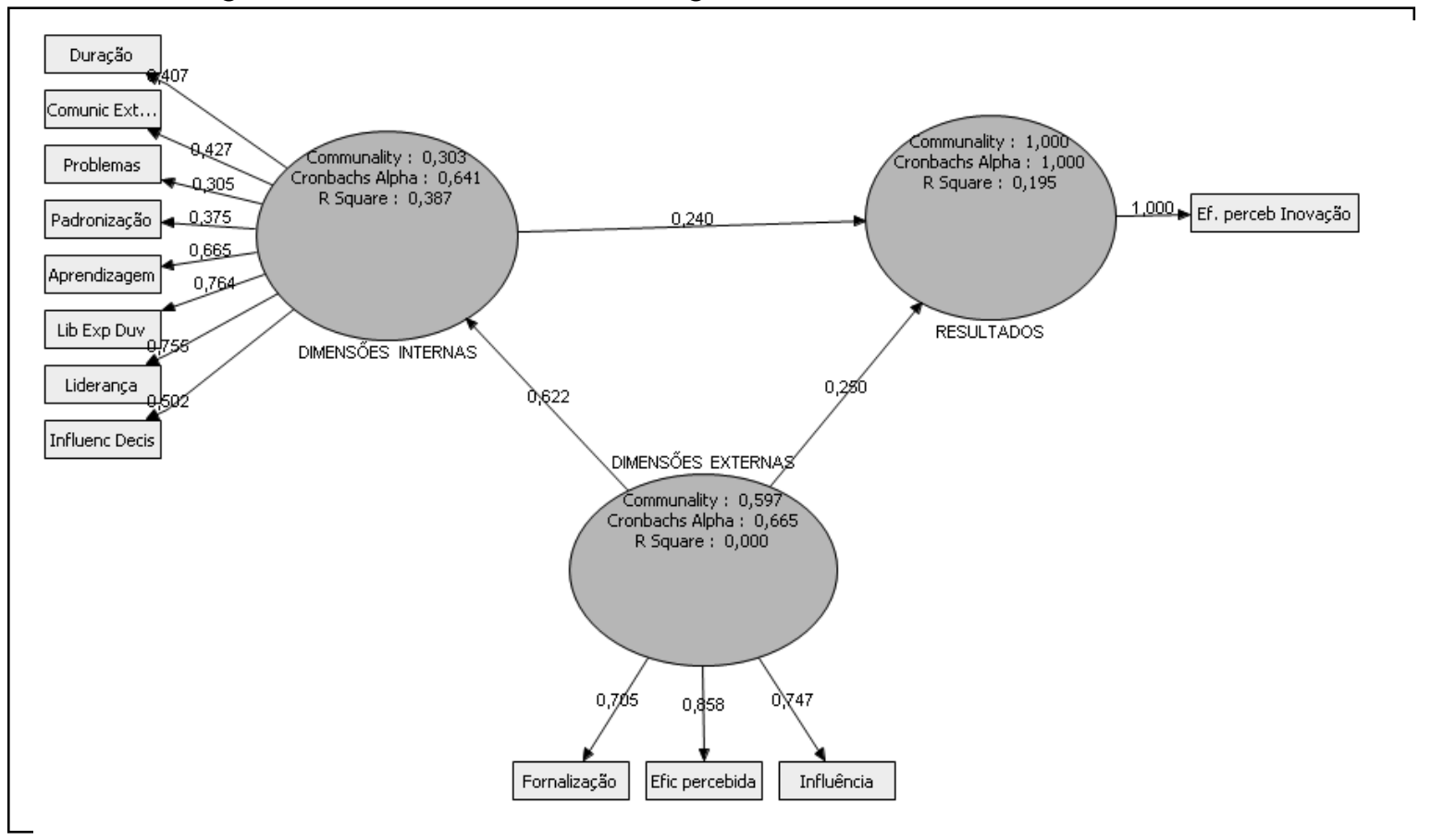

Fonte: Os Autores (2013)

A média das dimensões internas em conjunto apresenta um alfa de Cronbach satisfatório (64\%), mas as dimensões apresentam baixa comunalidade entre elas e impactam apenas $24 \%$ na percepção de eficiência na inovação. Neste grupo, podese destacar a liberdade para expressar dúvidas como característica marcante no ambiente interno da organização. Nesta unidade, ainda se pode afirmar que a estrutura adaptada possui uma correlação fraca sobre os resultados ou que o modelo proposto não pode ser evidenciado de forma significativa na unidade em estudo.

Já a unidade de Goiás apresenta um cenário diferenciado no que diz respeito à correlação entre as dimensões da estrutura. Ressalta-se que a confiabilidade dos dados nesta unidade apresenta índice superior à unidade do Rio Grande do Sul.

Conforme a Figura 3, as médias das dimensões externas são mais correlatas entre elas e apresentam um grau de confiabilidade das respostas superior a $70 \%$. CARVALHO, L. C.; MACHADO, D.D.P.N. Ambiente de Inovação: estudo comparativo entre três unidades de uma organização do setor metal-mecânico. Revista de

Empreendedorismo e Gestão de Pequenas Empresas, v. 2, n.1, p. 47-76, 2013. 
Assim como na filial do Rio Grande do Sul, a satisfação de parceria com outros grupos ligados a inovação pode ser percebida (dimensão 12). Destaca-se que o impacto destas dimensões sobre as internas é superior a $78 \%$ e que estas dimensões explicam as internas em 61\%. Já o impacto deste grupo sobre os resultados não pode ser verificado de forma significativa, ficando abaixo dos $20 \%$.

Figura 3: Estrutura da Metodologia MIS na Unidade de Goiás.

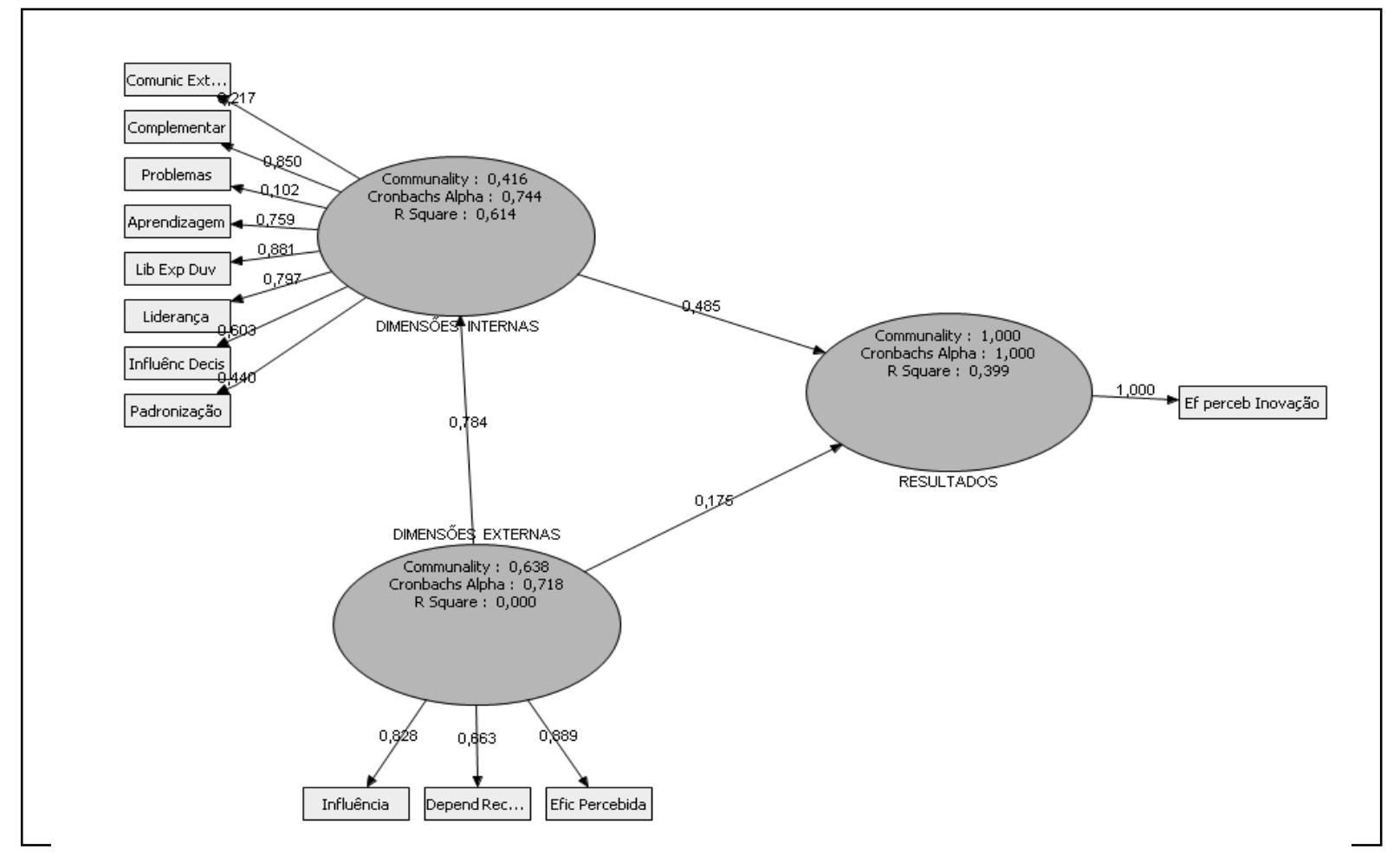

Fonte: Os Autores (2013).

As médias das dimensões internas apresentam um grau de confiabilidade das respostas maior (74\%) do que observado nas dimensões externas. E assim como na filial do Rio Grande do Sul, a liberdade para expressar dúvidas (dimensão 8) é atribuída a alta carga fatorial em relação às demais dimensões externas, sendo possível caracterizá-la como elemento formador do ambiente inovador nesta unidade. O impacto desta dimensão sobre os resultados é mais representativo do que o percebido na unidade do Rio Grande do Sul, chegando próximo aos 50\%. 
De acordo com a percepção dos funcionários, os resultados percebidos pela organização podem ser explicados em quase $40 \%$ pela covariância entre dimensões internas e externas. Portanto, afirma-se que esta estrutura ajusta-se ao ambiente da unidade de Goiás por apresentar índices satisfatórios de correlação.

Já a Figura 4 demonstra as relações entre as dimensões percebidas no ambiente da unidade de São Paulo. Nela, pode-se notar que as dimensões externas são percebidas semelhantemente às unidades do Sul e do Centro-Oeste brasileiro, e que a eficiência percebida por meio dos relacionamentos entre os grupos de inovação configura-se uma verdade.

As dimensões externas apresentam correlação média-alta com as dimensões internas e explicam as variações do ambiente interno em 47,5\%. O mesmo impacto dessas dimensões não pode ser evidenciado diretamente sobre os resultados, uma vez que a correlação está abaixo de $6 \%$.

Figura 4: Estrutura da Metodologia MIS na Unidade de São Paulo.

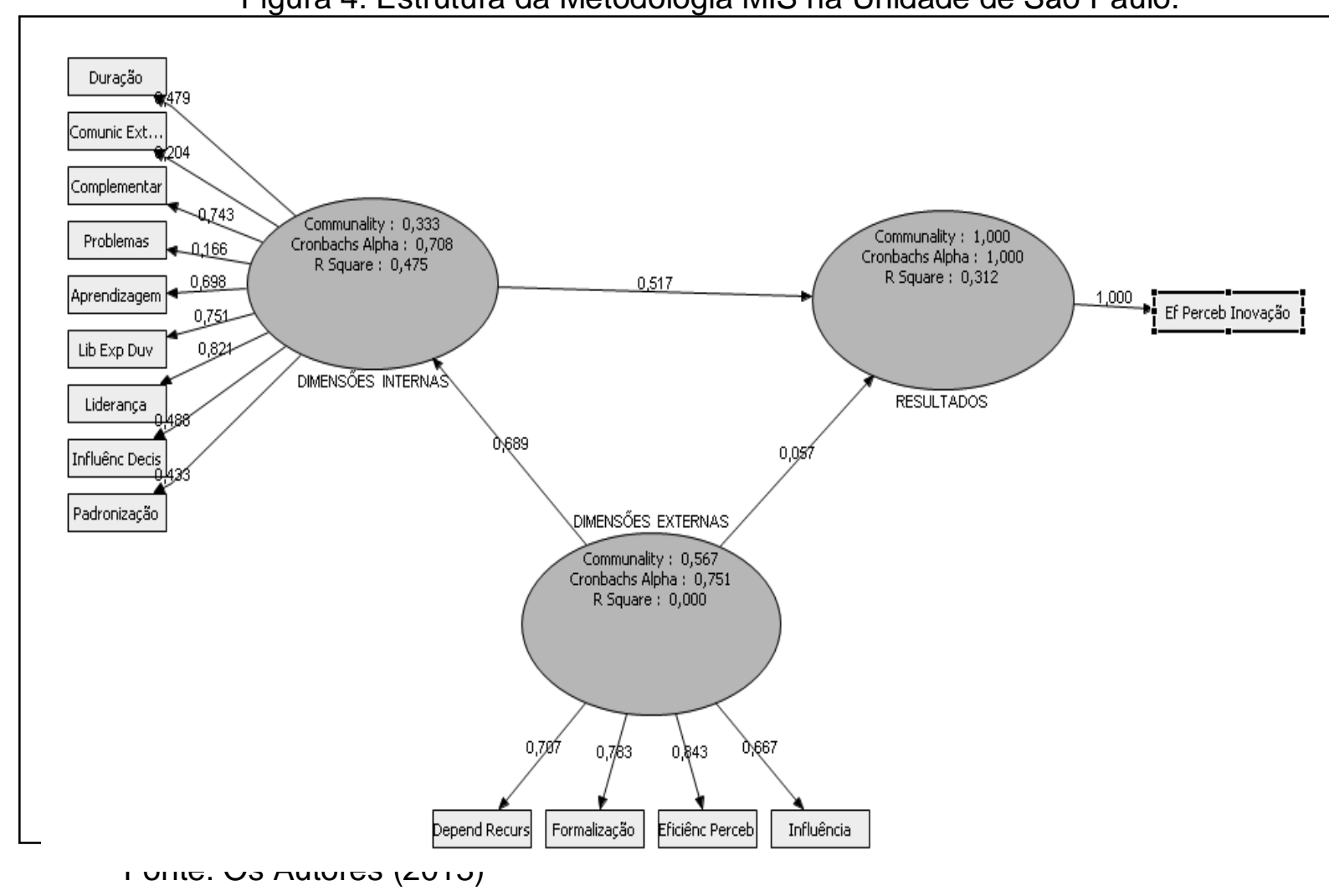

CARVALHO, L. C.; MACHADO, D.D.P.N. Ambiente de Inovação: estudo comparativo entre três unidades de uma organização do setor metal-mecânico. Revista de

Empreendedorismo e Gestão de Pequenas Empresas, v. 2, n.1, p. 47-76, 2013. 
As dimensões internas constantes no modelo proposto e percebidas pelos funcionários têm comunalidade baixa entre elas, mas possuem graus de confiabilidade aceitáveis. Neste grupo, as dimensões voltadas às ações da liderança e à liberdade para expressar dúvidas, destaca-se na percepção dos funcionários. Em termos de correlação, as dimensões internas impactam os resultados em $51,7 \%$, sendo o maior entre as três filiais.

Apesar de este modelo apresentar correlação satisfatória entre os grupos de dimensões, o poder de explicação destas sobre os resultados ainda é baixo $(31,2 \%)$.

\section{Comparação Entre as Unidades de Pesquisa}

A fim de verificar as diferenças e semelhanças entre os ambientes inovadores das organizações em estudo, apresenta-se um quadro comparativo para que possam ser observados os pontos relevantes e de destaque. A distinção dos pontos entre as unidades podem dar subsídios para a caracterização da unidade que, segundo a metodologia MIS, seja a mais inovadora. 
Tabela 7: Comparativo entre as Unidades Pesquisadas.

\begin{tabular}{|c|c|c|c|}
\hline & \multicolumn{3}{|c|}{ CONFIABILIDADE DAS RESPOSTAS } \\
\hline & Rio Grande do Sul & Goiás & São Paulo \\
\hline \multirow[t]{3}{*}{$\begin{array}{c}\text { Alfa de } \\
\text { Cronbach }\end{array}$} & $87,2 \%$ & $92,4 \%$ & $92,6 \%$ \\
\hline & DIMENSÕES PERCEBID & & \\
\hline & Rio Grande do Sul & Goiás & São Paulo \\
\hline \multirow[t]{3}{*}{ 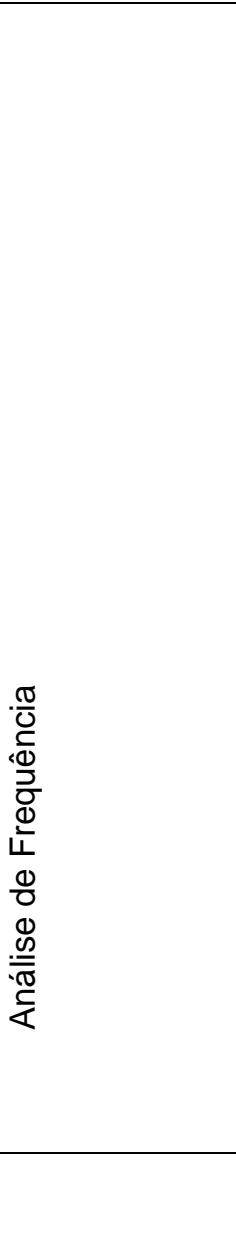 } & $\begin{array}{l}\text { Eficiência percebida com } \\
\text { a inovação (D1); } \\
\text { Padronização de } \\
\text { procedimentos (D4); } \\
\text { Grau de influência nas } \\
\text { decisões (D5); } \\
\text { Liderança do time de } \\
\text { inovação (D7); } \\
\text { Liberdade para } \\
\text { expressar dúvidas (D8); } \\
\text { Aprendizagem } \\
\text { encorajada (D9); } \\
\text { Formalização (D11); } \\
\text { Eficiência percebida } \\
\text { (D12); } \\
\text { Influência (D13); } \\
\text { Problemas Identificados } \\
\text { (D23); } \\
\text { Frequência (D28); de } \\
\text { comunicação (D2); } \\
\text { Duração (D29) }\end{array}$ & $\begin{array}{l}\text { Eficiência percebida com } \\
\text { a inovação (D1); } \\
\text { Padronização de } \\
\text { procedimentos (D4); } \\
\text { Grau de influência nas } \\
\text { decisões (D5); } \\
\text { Liderança do time de } \\
\text { inovação (D7); } \\
\text { Liberdade para } \\
\text { expressar dúvidas (D8); } \\
\text { Aprendizagem } \\
\text { encorajada (D9); } \\
\text { Dependência } \\
\text { recursos (D10); } \\
\text { Eficiência percebida } \\
\text { (D12); } \\
\text { Influência (D13); } \\
\text { Problemas Identificados } \\
\text { (D23); } \\
\text { Complementaridade } \\
\text { (D26); } \\
\text { Frequência } \\
\text { comunicação (D28); }\end{array}$ & $\begin{array}{l}\text { Eficiência percebida com } \\
\text { a inovação (D1); } \\
\text { Padronização } \\
\text { procedimentos (D4); } \\
\text { Grau de influência nas } \\
\text { decisões (D5); } \\
\text { Liderança do time de } \\
\text { inovação (D7); } \\
\text { Liberdade para } \\
\text { expressar dúvidas (D8); } \\
\text { Aprendizagem } \\
\text { encorajada (D9); } \\
\text { Dependência } \\
\text { recursos (D10); } \\
\text { Formalização (D11); } \\
\text { Eficiência percebida } \\
\text { (D12); } \\
\text { Influência (D13); } \\
\text { Problemas Identificados } \\
\text { (D23); } \\
\text { Complementaridade } \\
\text { (D26); } \\
\text { Frequência } \\
\text { comunicação (D28); de } \\
\text { Duração (D29). }\end{array}$ \\
\hline & \multicolumn{3}{|c|}{ DIMENSÕES CARACTERIZADORAS } \\
\hline & Rio Grande do Sul & Goiás & São Paulo \\
\hline \multirow[t]{3}{*}{ 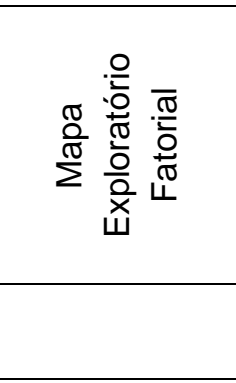 } & $\begin{array}{l}\text { F1: Liberdade para } \\
\text { expressar dúvidas (D8); } \\
\text { F2: Grau de influência } \\
\text { nas decisões (D5); } \\
\text { F3: Duração (D29) }\end{array}$ & $\begin{array}{l}\text { F1: Liderança do time de } \\
\text { inovação (D7); } \\
\text { F2: Grau de influência } \\
\text { nas decisões (D5); } \\
\text { F3: Problemas } \\
\text { Identificados (D23) }\end{array}$ & $\begin{array}{l}\text { F1: Liberdade para } \\
\text { expressar dúvidas (D8); } \\
\text { F2: Grau de influência } \\
\text { nas decisões (D5); } \\
\text { F3: Frequência de } \\
\text { comunicação (D28) }\end{array}$ \\
\hline & \multicolumn{3}{|c|}{ ADEQUAÇÃO DO AMBIENTE À ESTRUTURA DA METODOLOGIA MIS } \\
\hline & Rio Grande do Sul & Goiás & São Paulo \\
\hline 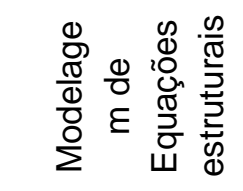 & $19,5 \%$ & $39,9 \%$ & $31,2 \%$ \\
\hline
\end{tabular}

Fonte: Os Autores (2013)

CARVALHO, L. C.; MACHADO, D.D.P.N. Ambiente de Inovação: estudo comparativo entre três unidades de uma organização do setor metal-mecânico. Revista de

Empreendedorismo e Gestão de Pequenas Empresas, v. 2, n.1, p. 47-76, 2013. 
Por meio das modelagens de equações estruturais procurou-se verificar se a estrutura do processo de inovação proposta pelo MIRP pode ser evidenciada nas unidades. Desta forma, pode-se constatar que a unidade de Goiás foi a que obteve melhor adequação ao modelo (39,9\%) por impetrar, por meio da estrutura proposta, maior poder de explicação à percepção de eficiência da inovação. Já a filial do Rio Grande do Sul, dentre as três unidades, foi a que obteve menor grau de explicação do modelo. De acordo com a metodologia MIS, afirma-se que a unidade mais inovadora é a filial de Goiás, seguida pela unidade de São Paulo e por último, a unidade do Rio Grande do Sul.

\section{Considerações Finais}

A presente pesquisa teve como direcionamento a verificação dos ambientes inovadores nas três unidades da empresa Alpha dispersas geograficamente nos estados do Rio Grande do Sul, Goiás e São Paulo. Para esta verificação, foi utilizado um questionário adaptado do MIRP, desenvolvido sob uma metodologia denominada MIS que realiza testes psicométricos quanto à existência de dimensões constantes de um ambiente inovador. O questionário adaptado contou com 21 dimensões abrangendo questões relacionadas a resultados e ambientes interno e externo das organizações.

Destas 21 dimensões, 12, 12 e 14 dimensões foram percebidas nas unidades do Rio Grande do Sul, Goiás e São Paulo, respectivamente. Das dimensões percebidas, 10 são comuns entre elas e estão todas relacionadas às ações da liderança voltadas ao desenvolvimento de inovações. Isto é: o grau com que as pessoas percebem a inovação, padronização das regras e detalhamento das mesmas para a execução das tarefas, percepção de influência na definição dos objetivos da organização, ações da liderança claras e bem definidas, liberdade para expressar dúvidas, aprendizagem encorajada pelos líderes, percepção de eficiência no relacionamento com outros grupos de inovação, grau de influência das equipes de inovação sobre as demais, percepção de ausência de barreiras para desenvolvimento da inovação. 
Registou- se comunalidade entre as unidades na percepção de não existência de 7 dimensões, que são: incerteza sobre a inovação, escassez de recursos, expectativas de prêmios e sanções, frequência de com que os membros do grupo de inovação se comunicam dentro e fora da organização, existência de conflitos, processo de resolução de conflitos e frequência de conflitos. Em suma, das 21 dimensões em estudo, 17 tiveram percepções comuns, positivas ou negativas, entre as unidades.

Pode-se notar, nesse sentido, que as políticas voltadas ao gerenciamento das três unidades de pesquisa possuem um grau de uniformidade, trabalhadas e repassadas aos funcionários de forma aberta, propiciando o correto entendimento e fazendo com que a haja percepções semelhantes das dimensões do ambiente interno nas três organizações.

Portanto, de uma forma geral, é prudente afirmar que as três unidades estudadas possuem ambientes de inovação semelhantes, diferenciadas por no máximo 2 dimensões peculiares a cada organização e pelo grau de percepção das dimensões.

Em última análise, verificou-se se a estrutura proposta pelo MIRP, adaptada para este estudo conforme a Figura 1 pode ser evidenciada nas três unidades. Com base nos dados apresentados, entende-se que a filial de Goiás apresenta melhores percepções de ambientes voltados a inovação, apontando índice próximo a 40\% para a definição das dimensões internas e externas como responsáveis pela percepção de eficiência da inovação. Concluiu-se, de acordo com a metodologia MIS, a unidade de Goiás parece ser a mais inovadora entre as unidades pesquisadas.

CARVALHO, L. C.; MACHADO, D.D.P.N. Ambiente de Inovação: estudo comparativo entre três unidades de uma organização do setor metal-mecânico. Revista de

Empreendedorismo e Gestão de Pequenas Empresas, v. 2, n.1, p. 47-76, 2013. 


\section{Referências}

AFUAH, A. La dinâmica de la innovación organizacional: el nuevo concepto para lograr ventajas competitivas y rentabilidad. México: Oxford University Press, 1999.

ALVARES, A. C. T. et al. Análise comparativa entre dois casos e considerações finais. In: BARBIERI, J.C. (Org). Organizações Inovadoras: estudos e casos brasileiros. Rio de Janeiro: FGV, 2003.

ANDRADE, T. Inovação e Ciências Sociais: em busca de novos referenciais. Revista Brasileira de Ciências Sociais, v. 20, n. 58, p. 145-211, 2005.

BARBETTA, P. A. Estatística aplicada às ciências sociais. Florianópolis: Ed. UFSC, 2001.

BARBIERI, J. C. et al. Organizações inovadoras: estudos e casos brasileiros. 2. ed. Rio de Janeiro: FGV, 2003.

BARBIERI, J. C.; ALVARES, A. C. T. O retorno dos sistemas de sugestão: abordagens, objetivos e um estudo de caso. Rio de Janeiro: Cadernos EBAPE. Edição Especial, 2005.

DAFT, R. L. Bureaucratic versus nonbureaucratic structure and process of innovation and change. In: BACHARACH, S. B. (Ed.) Research in the sociology of organizations. p. 129-166. Greenwich, CT: JAI Press, 1982.

DAMANPOUR, F. Organizational Size and Innovation. Organization Studies. p. 375-402, 1992. 
DAMANPOUR, F.; EVAN, W. M. Organizational innovation and performance: the problem of organizational lag. Administrative Science Quarterly, v. 29, p. 392-409, 1984.

FAGERBERG, J.; SRHOLEC, M.; KNELL, M. The Competitiveness of Nations: Why Some Countries Prosper While Others Fall Behind. World Development, v. 35, p. 1595-1620, 2007.

GRANT, R. M. Toward a Knowledge-Based Theory of the Firm. Strategic Management Journal, v. 17, p. 109-22, 1996.

HAIR JR, J. F. et al. Análise multivariada de dados. 5. ed. Porto Alegre: Bookman, 2005.

JONASH, R. S.; SOMMERLATTE, T. O valor da inovação: como as empresas avançadas atingem alto desempenho. Rio de Janeiro: Campus, 2001.

KNOX, S. The boardroom agenda: developing the innovative organization.

Corporate Governance. v. 2, n. 1, Bradford, UK, p. 27-36, 2002.

LAM, Alice. Organizational Innovation. In: FAGERBERG, J; MOWERY, D. C.; NELSON, Richard R. (Orgs). The Oxford Handbook of Innovation. Oxford: Oxford University Press, 2005. p. 116-47.

MACIEL, M. L. Inovação e conhecimento. In: SOBRAL, F. et al. (Orgs.). A alavanca de Arquimedes: ciência e tecnologia na virada do século. Brasília: Paralelo 15, 1997.

MACHADO, D. D. P. N. Inovação e cultura organizacional: um estudo dos elementos culturais que fazem parte de um ambiente inovador. 2004. 185p. Tese 
(Doutorado em Administração de Empresas) - Escola de Administração de Empresas de São Paulo da Fundação Getúlio Vargas. São Paulo, 2004.

OCDE- Organisation for Economic Co-Opperation and Developmet e FINEP Financiadora de Estudos e Projetos. Manual de Oslo: diretrizes para coleta e interpretação de dados sobre inovação. 3. ed. Brasília, 2005.

RICYT - Red Iberoamericana de Indicadores de Ciencia y Tecnología e OEA Organización de Estados Americanos. Manual de Bogotá. 2001.

SCHUMPETER, J. Capitalism, Socialism, and Democracy. New York: Harper, 1942.

SIMON, H. A. Bounded rationality and Organizational Learning. Organization Science, v. 2, p. 125-34, 1991.

TERRA, J. C. C. Gestão do Conhecimento: aspectos conceituais e estudo exploratório sobre as práticas de empresas brasileiras. Tese (Doutorado em Engenharia da Produção). Departamento de Engenharia de Produção da Escola Politécnica da Universidade de São Paulo, 1999.

TIDD, J.; BESSANT, J.; PAVITT, K. Managing innovation integrating technological, market and organizational change. New York: John Wiley \& Sons, 1997.

TORNATSKY, L. G. et al. The process of technological innovation: Reviewing the Literature. In: National Science Foundation. Washington, 1983. p. 17.

VAN DE VEN, A. H.; CHU, Y. A psychometric assessment of the Minnesota innovation survey. Chapter 3. In: VAN DE VEN, A.H;POOLE, M. S.(eds). Research 
on the management of innovation. The Minnesota Studies, New York:

Ballinger/Harper \& Row, 1989.

VAN DE VEN, A.H.; POOLE, M. S. Methods for studying innovation. Development in the Minnesota Innovation Research Program. Organizational Science, v. 1, n. 3, p. 313-35, 1990.

VICENTI, T. Ambiente de inovação nas empresas de software de Blumenau Santa Catarina - Brasil. 2006. 183p. Dissertação (Mestrado em Administração de Empresas) - Fundação Universidade Regional de Blumenau - Blumenau: FURB, 2006.

VIEIRA, V. A. As tipologias, variações e características da pesquisa de marketing. Revista da FAE, v. 5, n. 1, p. 61-70, jan/abr. 2002.

Artigo recebido em: 11/03/2013 Aprovado em 30/04/2013. 